\title{
Short-term outcomes of benign paroxysmal positional vertigo with and without sudden sensorineural hearing loss: a retrospective study
}

\author{
Hainan $\mathrm{Cao}^{1}$, Xian $\mathrm{Gao}^{1}$, Jun $\mathrm{Su}^{2}$, Haiping Duan ${ }^{3}$, and Wen Jiang ${ }^{4}$ \\ ${ }^{1}$ Qingdao Municipal Hospital Group \\ ${ }^{2}$ Zhejiang Chinese Medical University \\ ${ }^{3}$ Qingdao Municipal Center for Disease Control and Prevention \\ ${ }^{4}$ Xuzhou Medical University
}

August 27, 2020

\begin{abstract}
Objective: This study aimed to investigate the outcome of adults with benign paroxysmal positional vertigo(BPPV)secondary to sudden sensorineural hearing loss(SSNHL). Methods: A retrospective study was conducted in patients with evidence of idiopathic BPPV or SSNHL with BPPV . All participants were identified and categorized by using the Dix-Hallpike test and roll test, followed by canalith repositioning procedures (CRPs) that were repeated 2 to 3 times. Assessment of the outcome was conducted one-hour and one-week post-CRPs, respectively. Results Six-four adults with BPPV secondary to SSNHL and 328 adults with idiopathic BPPV were included. The posterior canal was the most affected in both groups. At one-hour posttreatment, the cured rate of the BPPV with SSNHL group was $45.31 \%$, which was significantly lower than that of the iBPPV group $(94.82 \%, \mathrm{P}<0.01)$. At one-week posttreatment, the cured rate of the BPPV with SSNHL group was $66.67 \%$, which was significantly lower than that of the i-BPPV group $(98.78 \%, \mathrm{P}<0.01)$. Conclusions BPPV with SSNHL was associated with poorer outcomes than i-BPPV in the short term.
\end{abstract}

Short-term outcomes of benign paroxysmal positional vertigo with and without sudden sensorineural hearing loss: a retrospective study

Abstract

Objectives: This study aimed to investigate the outcome of adults with benign paroxysmal positional vertigo(BPPV)secondary to sudden sensorineural hearing loss(SSNHL).

Design: Retrospective study

Setting: removed for blind peer review

Participants: Six-four adults with BPPV secondary to SSNHL and 328 adults with idiopathic BPPV were included.

Main outcome measures: All participants were identified and categorized by using the Dix-Hallpike test and roll test, followed by canalith repositioning procedures (CRPs) that were repeated 2 to 3 times. Assessment of the outcome was conducted one-hour and one-week post-CRPs, respectively.

Results: The posterior canal was the most affected in both groups. At one-hour posttreatment, the cured rate of the BPPV with SSNHL group was $45.31 \%$, which was significantly lower than that of the i-BPPV group $(94.82 \%, \mathrm{P}<0.01)$. At one-week posttreatment, the cured rate of the BPPV with SSNHL group was 
$66.67 \%$, which was significantly lower than that of the i-BPPV group $(98.78 \%, \mathrm{P}<0.01)$. In the BPPV with SSNHL group, the cured rate of 1 hour post-CRPs was 45.31\%, which was significantly lower than that of 1 week post-CRPs $(65.63 \%, \mathrm{P}<0.05)$.

Conclusions: BPPV with SSNHL was associated with poorer outcomes than i-BPPV in the short term.

Key words:Benign paroxysmal positional vertigo, Sudden sensorineural hearing loss, Outcome

Keypoints

1.We retrospectively analysed the effectiveness of CRPs in SSNHL and i-BPPV patients.

2. All patients received sufficient CRPs, and the cured rate was an indicator of their effectiveness.

3.Sufficient CRPs meant that during one outpatient service, we perform CRPs unti typical positional nystagmus disappeared. If the symptoms remained, we performed CRPs a maximum of 3 times.

4. The BPPV with SSNHL group was associated with poorer outcomes than the i-BPPV group 1hour and 1week post-CRPs.

5. The cured rate was significantly improved without repeated CRPs after one week of medication in the BPPV with SSNHL group, which suggested that repeated CRPs might not be necessary for patients with poor effectiveness of BPPV with SSNHL.

\section{Introduction}

The prevalence of sudden sensorineural hearing loss (SSNHL) ranges from 5 to 27 per 100,000 people ${ }^{1}$. Meanwhile, the prevalence of benign paroxysmal positional vertigo (BPPV) is 10.7 140 per 100,000 people annually $^{2}$. Vertigo is reported in 30 to $40 \%$ of SSNHL cases and leads to the poor prognosis of SSNHL ${ }^{3,4}$, while 8.8 to $12.7 \%$ of patients with SSNHL present with BPPV ${ }^{5}$. SSNHL with vertigo occurs in many kinds of specific disease conditions, and BPPV is the most common type of vertigo, and can be effectively treated by canalith repositioning procedures $(\mathrm{CRPs})^{2,6}$. However, some studies have shown that BPPV with SSNHL has different clinical characteristics from idiopathic BPPV (i-BPPV $)^{7,8}$. BPPV with SSNHL is very problematic, and it has a prolonged course ${ }^{9}$. In addition to these characteristics, on caloric testing, more canal paresis was present in the BPPV with SSNHL group ${ }^{10}$. Recent studies on BPPV with SSNHL focused on the effectiveness of CRPs by comparing the number of CRPs, and these studies treated BPPV with CRPs and improved hearing loss by medication at the same time ${ }^{3,7,10,11}$. We found that it took longer to achieve successful repositioning, and these studies ignored the effects of medicines on the duration of treatment. This study aimed to review the clinical characteristics of adults with BPPV and SSNHL and to compare them with those of i-BPPV adults. Moreover, we explored the effectiveness of medication treatment without repeated CRPs.

2.Materials and Methods

\subsection{Participants}

We retrospectively analysed 64 patients with BPPV and SSNHL and 328 patients with i-BPPV between January 1, 2016, and February 26, 2019, at the Department of Otolaryngology, removed for blind peer review.

\subsection{Audiological Examinations}

Patients with BPPV secondary to SSNHL received additional measurements, specifically pure-tone audiometry(Madsen Astera)and tympanometry(Madsen Zodiac 901). Patients with SSNHL were divided into three types according to their hearing loss: mild and moderate hearing loss ( $\left.26^{\sim} 60 \mathrm{~dB} \mathrm{HL}\right)$, severe hearing $\operatorname{loss}(60 \sim 80 \mathrm{~dB} \mathrm{HL})$, or profound hearing loss (>80 dB HL).[12]

2.3 BPPV Specific Assessments 
All patients completed a medical history and neurotologic test, including video electronystamography (ICS Chartr 200) and positioning tests (such as the Dix-Hallpike test and the roll test). We divided patients into two groups:the i-BPPV group and the BPPV with SSNHL group. The clinical characteristics of i-BPPV are typical BPPV-related nystagmus without inner ear disease or any other abnormal neurotologic findings ${ }^{13}$. The patients had unilateral sensorineural hearing loss(SNHL) that occurred suddenly within a 72-hour window, and the audiometric criterion was a decrease in hearing greater than or equal to $20 \mathrm{~dB}$, that affected at least two consecutive frequencies ${ }^{14}$. Patients with additional established diseases, such as Meniere's disease, labyrinthitis, vestibular neuritis, vestibular migraine, superior semicircular canal dehiscence syndrome, posterior circulation ischaemia, postural hypotension, and central paroxysmal positional vertigo, were excluded from this study.

The involved semicircular canal was confirmed by positioning tests in all patients, which determined the manoeuvres used in canalith repositioning. If the typical positional nystagmus did not disappear or decrease after CRP, we would perform sufficient CRPs, up to maximum of 3 times $^{15}$. All patients with SSNHL had medication according to the clinical practice guidelines: sudden sensorineural hearing loss (2015) by the Chinese Academy of Otolaryngology-Head and Neck Surgery(The guidelines referred to the German guidelines for sudden idiopathic sensorineural hearing loss (2004) and the USA guidelines for sudden hearing $\operatorname{loss}(2012))$.

The outcome used two grades: cured and uncured, which were evaluated at 1 hour and 1 week after the initial CRPs.

\subsection{Statistical analysis}

We employed statistics for independent groups with a threshold of $p<0.05$, using SPSS statistical software for Windows, version 21.0 (IBM, Armonk, NY). $\chi^{2}$ tests were used to determine the association between categorical variables. Statistical significance was set at $p<0.05$.

\section{Results}

3.1 Comparison of effectiveness between two groups at 1hour post-CRPs and 1week post-CRPs

Based on the inclusion criteria, we recruited 64 patients with BPPV secondary to SSNHL and 328 patients with i-BPPV (Table 1). The two groups were both slightly female dominant, and sex distributions were not different between the BPPV with SSNHL and i-BPPV groups. However, the age of the two groups was significantly different $(p<0.001)$. The percentage of patients with profound hearing loss was the highest, and patients with profound or severe hearing loss made up the majority (Figure 1). Comparing the effectiveness of CRPs 1 hour after treatment by $\chi^{2}$ test(Fig 2), there was a significant difference between the BPPV with SSNHL group and the i-BPPV group $(p<0.001)$. Comparing the effectiveness of CRPs 1 week after treatment by Fisher's exact test (Figue 3), there was a significant difference between the BPPV with SSNHL group and the i-BPPV group $(p<0.001)$.

\subsection{The effectiveness of CRPs at 1 hour and 1 week after treatment}

Comparing the effectiveness of CRPs 1 hour and 1week after treatment with CRPs with a $\chi^{2}$ test for trends, there was a significant difference $(p=0.021)$, (Figure 4$)$.

\section{Discussion}

SSNHL is often associated with vertigo, and BPPV is one of the common causes of vertigo in patients with $\mathrm{SSNHL}^{9}$. BPPV is due to the detachment of the otolith from the utricle, which can be effectively treated by canalith repositioning procedures $(\mathrm{CRPs})^{6}$. However, the cause of detachment is still unclear. In our study, we found that most of the patients in the BPPV with SSNHL group had severe or profound hearing loss, which was consistent with published studies ${ }^{10,16,17}$. SSNHL with severe or profound hearing loss is often associated with vascular causes ${ }^{13}$. This suggests that the aetiology and pathophysiology of BPPV with SSNHL is mainly related to vascular factors. 
In our study, there was no difference between the two groups in terms of sex composition or involved semicircular canal type, only in terms of age. Junghee Yoon reported that age $>50$ years was one of the risk factors associated with the effectiveness of $\mathrm{CRPs}^{18}$. However, although the mean age of the BPPV with SSNHL group was younger than 50 years and that of the i-BPPV group was older than 50 years, the effectiveness of CRPs in the BPPV with SSNHL group was worse than that in the i-BPPV group. Other studies have also shown that BPPV with SSNHL is often intractable ${ }^{7,8,10}$. Although we found that the CRPs were valid for the BPPV with SSNHL and i-BPPV groups, the effectiveness of the CRPs in the BPPV with SSNHL group was significantly worse than that in the i-BPPV group. This result suggested that BPPV with SSNHL was caused not only by the detachment of otoliths but also by other reasons. Some studies might help explain this phenomenon. Kim(2014) showed that blood debris in the endolymph due to inner ear haemorrhage was one of the causes of BPPV in patients with ipsilateral SSNHL ${ }^{19}$, and CRPs were ineffective in this kind of patient. Gacek (2003) proposed a pathophysiological mechanism for BPPV that included loss of the inhibitory effect of the otolith organs on the canal sense organs by studying five temporal bones from patients with BPPV, and atlthough these patients had symptoms of BPPV , there was no detachment of the otoliths $^{20}$. Both of the abovementioned hypotheses could lead to the failure of CRPs. Therefore, we deduced that the above multiple reasons caused BPPV with SSNHL.

Studies have shown that the number of CRPs needed to treat BPPV with SSNHL was greater than that needed to treat i-BPPV ${ }^{3,10,16}$. In these studies, CRPs were usually conducted every 2 to 3 days, which meant that repeated CRPs eventually cured BPPV with SSNHL in one or two weeks. Moreover, upon completion of the treatment, all patients with SSNHL received medication for hearing loss. In our study, patients did not receive repeated CRPs between the two evaluations;they only received medication for hearing loss. We found that the cured rate was significantly improved without repeated CRPs after medication for one week in the BPPV with SSNHL group. We chose the cured rate rather than the effective rate for comparing the effectiveness of CRPs 1 hour and 1 week post treatment between two groups because the typical positional nystagmus(TPN) in many patients had decreased but not disappeared at 1 hour post-CRPs and TPN disappeared at 1 week post-CRPs. These results suggested that medication might be one of the choices that could improve the cured rate of intractable BPPV with SSNHL, similar to repeated CRPs.

\section{Conclusion}

The clinical characteristics of BPPV with SSNHL are similar to those of i-BPPV, while BPPV with SSNHL is associated with poorer outcomes than i-BPPV in the short term. The cured rate was significantly improved without repeated CRPs after 1 week of medication in the BPPV with SSNHL group. Thus, due to adverse reactions such as vertigo, vomiting, and hyperventilation during CRP, and its high cost, it might be costeffective to take medication after the initial CRPs and not repeating CRPs in the first week for BPPV with SSNHL.

\section{References}

1. Chandrasekhar, S. S., Tsai Do, B. S., Schwartz, S. R., Bontempo, L. J., Faucett, E. A., Finestone, S. A., . . . Monjur, T. M. (2019). Clinical Practice Guideline: Sudden Hearing Loss (Update) Executive Summary. Otolaryngol Head Neck Surg, 161(2), 195-210.

2. Bhattacharyya, N., Gubbels, S. P., Schwartz, S. R., Edlow, J. A., El-Kashlan, H., Fife, T., . . . Corrigan, M. D. (2017). Clinical Practice Guideline: Benign Paroxysmal Positional Vertigo (Update). OtolaryngologyHead and Neck Surgery, 156(3), 1-47.

3. Kim, M. B., \& Ban, J. H. (2012). Benign paroxysmal positional vertigo accompanied by sudden sensorineural hearing loss: a comparative study with idiopathic benign paroxysmal positional vertigo. Laryngoscope, 122(12), 2832-2836.

4. Park, S.-W. J. C.-K. R. H.-M. (2001). Vestibular Diagnosis as Prognostic Indicator in Sudden Hearing Loss with Vertigo. Acta Oto-Laryngologica, 121(533), 80-83. 
5. Hong, S. M., \& Yeo, S. G. (2013). Clinical analysis of patients with idiopathic sudden sensorineural hearing loss and benign paroxysmal positional vertigo. Acta Oto-Laryngologica, 133(5), 439-442.

6.Helminski, J. O., Zee, D. S., Janssen, I., \& Hain, T. C. (2011). Effectiveness of particle repositioning maneuvers in the treatment of benign paroxysmal positional vertigo: a systematic review. Physical Therapy, 90(5), 663-678.

7. Lee, N. H., Ban, J. H., Lee, K. C., \& Kim, S. M. (2010). Benign paroxysmal positional vertigo secondary to inner ear disease. Otolaryngol Head Neck Surg, 143(3), 413-417.

8. Luryi A L, Schutt C A, Bojrab D I, et al. Causes of Persistent Positional Vertigo Following Posterior Semicircular Canal Occlusion for Benign Paroxysmal Positional Vertigo[J]. Otology \& neurotology, 2018, 39(10):e1078-e1083.

9. von Brevern, M., Bertholon, P., Brandt, T., Fife, T., Imai, T., Nuti, D., \& Newman-Toker, D. (2015). Benign paroxysmal positional vertigo: Diagnostic criteria. J Vestib Res, 25(3-4), 105-117.

10. Lee, J. B., \& Choi, S. J. (2015). Canal Paresis in Benign Paroxysmal Positional Vertigo Secondary to Sudden Sensorineural Hearing Loss. 36(10)(1537-4505 (Electronic)), 1708-1713.

11. Zhang, H., Zhang, Q., Xu, D., \& Xu, M. (2014). Treatment outcome of patients with idiopathic sudden sensorineural hearing loss and concomitant benign paroxysmal positional vertigo. J Clin Otorhinolaryngol Head Neck Surg, 28(16), 3.

12.Qian, Y., Zhong, S. , Hu, G. , Kang, H. , Wang, L. , \& Lei, Y. . (2018). Sudden sensorineural hearing loss in children. Otology \& Neurotology, 39(8), 1018-1024.

13.Editorial Board of Otorhinolaryngology Head and Neck Surgery,\& Chinese Otorhinolaryngology Head Surgery Society of Chinese Medical Association. Guildlines for diagnosis and treatment of sudden sensorineural hearing loss (2015). Chinese Journal of Otolaryngology, 2015, 50(6):443-447.

14. Editorial Board of Otorhinolaryngology Head and Neck Surgery,\& Chinese Otorhinolaryngology Head Surgery Society of Chinese Medical Association. Guildlines for diagnosis and treatment of benign paroxysmal positional vertigo(2017).Chinese Journal of Otolaryngology, 2017, 52(3):173-177.

15. Brandt T, Dieterich M, StruppM. Vertigo and dizziness. 2nd ed. London : Springer, 2013.

16. Lee, N. H. ,\& Ban, J. H. . (2010). Is bppv a prognostic factor in idiopathic sudden sensory hearing loss?. Clinical \& Experimental Otorhinolaryngology, 3(4), 199-202.

17. Hong, S. M. ,\& Yeo, S. G. . (2013). Clinical analysis of patients with idiopathic sudden sensorineural hearing loss and benign paroxysmal positional vertigo. Acta Oto Laryngologica, 133(5), 439-442.

18. Yoon, J. , Lee, J. B. , Lee, H. Y. , Lee, B. D. , Lee, C. K. , \& Choi, S. J. . (2018). Potential risk factors affecting repeated canalith repositioning procedures in benign paroxysmal positional vertigo. Otology \& Neurotology, 39(2), 206-211.

19. Kim, C. H., Shin, J. E., Park, H. J., Koo, J. W., \& Lee, J. H. (2014). Concurrent posterior semicircular canal benign paroxysmal positional vertigo in patients with ipsilateral sudden sensorineural hearing loss: is it caused by otolith particles? Med Hypotheses, 82(4), 424-427.

20. Gacek, R. R. (2003). Pathology of benign paroxysmal positional vertigo revisited. Annals of Otology, Rhinology \& Laryngology, 112(7), 574-582.

\section{Hosted file}

Table 1 Characteristics of the Study Populations.docx available at https://authorea. com/users/353317/articles/477278-short-term-outcomes-of-benign-paroxysmal-positionalvertigo-with-and-without-sudden-sensorineural-hearing-loss-a-retrospective-study 


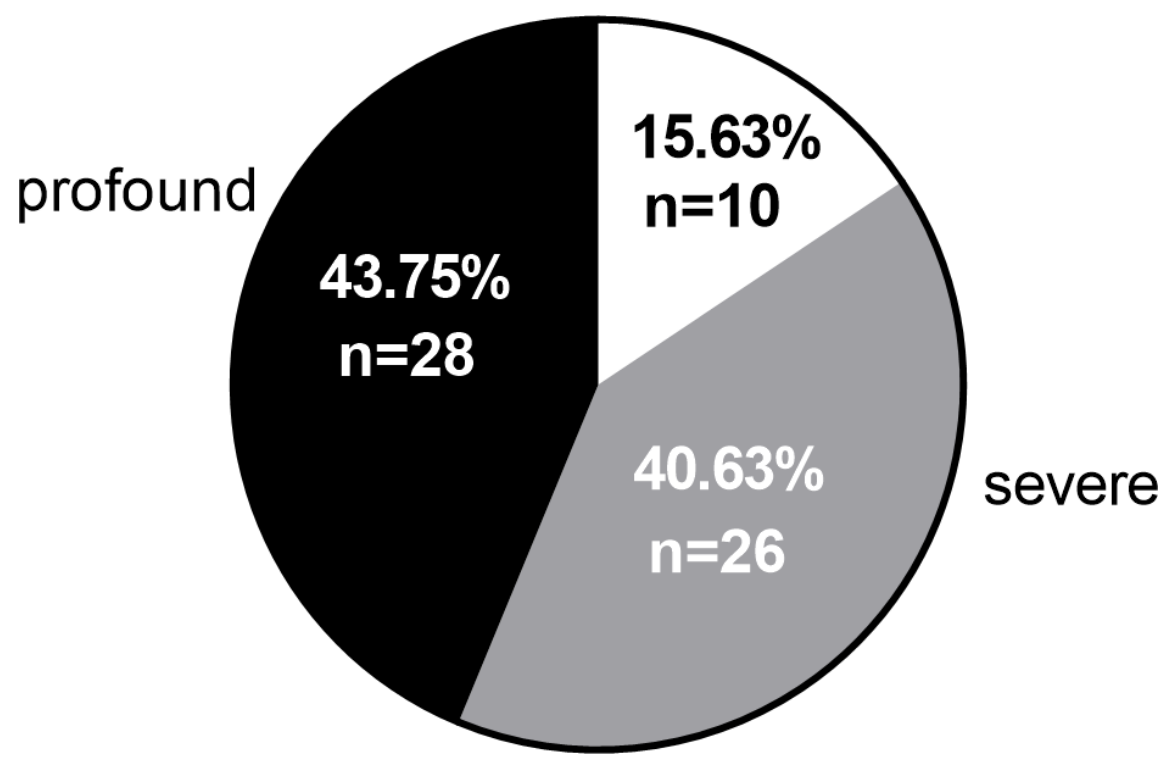

ए Cured $\boxminus$ Uncured

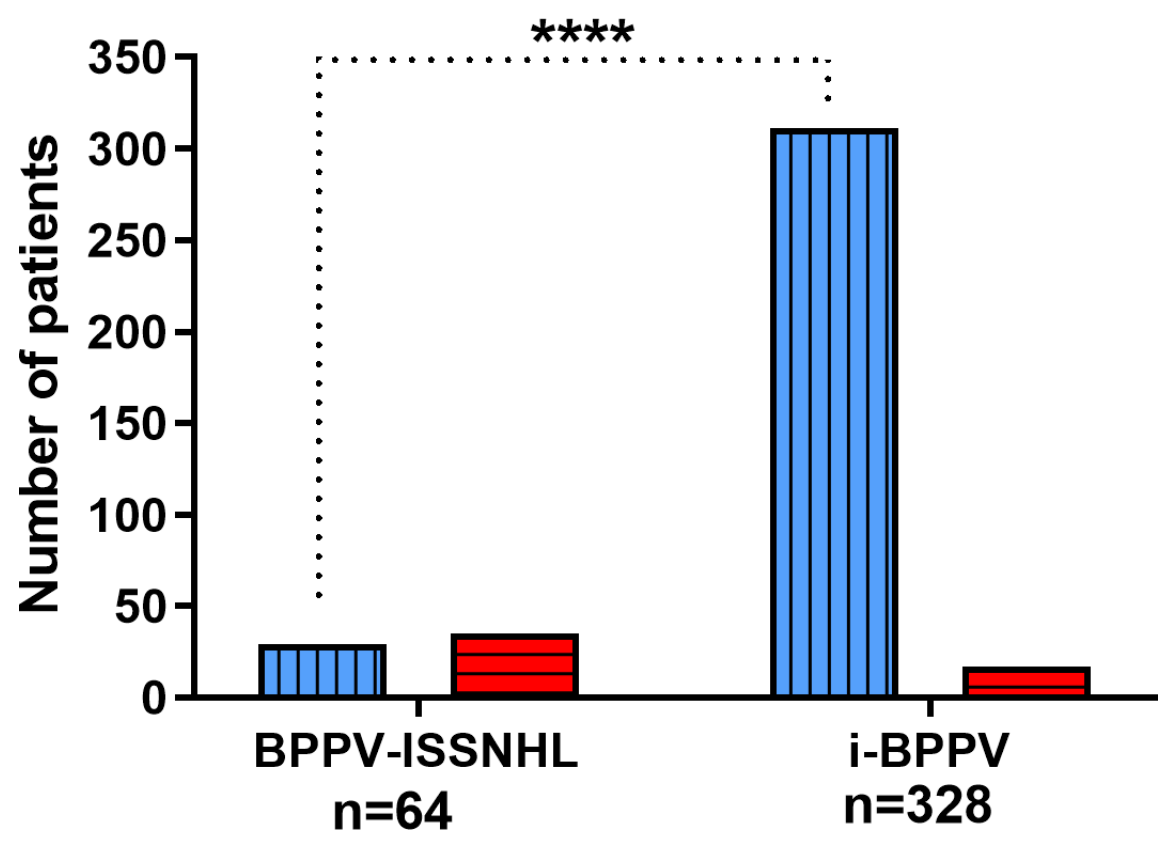



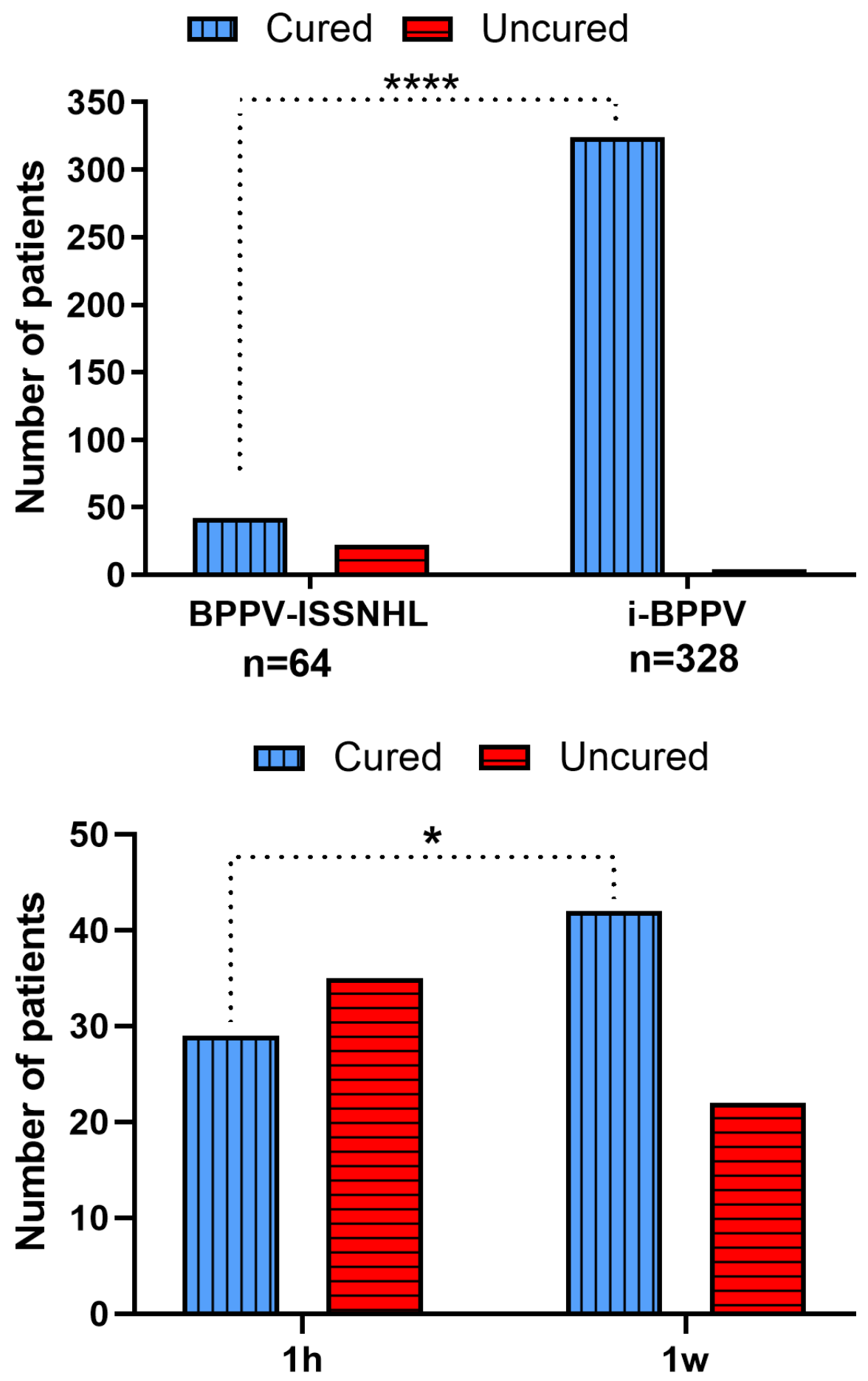